\title{
INFLUÊNCIA DE UM TRATAMENTO TÉRMICO DE RESSOLUBILIZAÇÃO ANTES DA LAMINAÇÃO DE UMA LIGA DE ALUMÍNIO 7475-T7351*
}

\author{
Saulo Brinco Diniz ${ }^{1}$ \\ Andrey de Moraes Barcelos Casanova ${ }^{2}$ \\ Patrícia Freitas Rodrigues ${ }^{3}$ \\ Andersan dos Santos Paula ${ }^{4}$ \\ Luiz Paulo Mendonça Brandão ${ }^{5}$
}

\section{Resumo}

O presente trabalho buscou explorar a influência de um tratamento térmico de ressolubilização $\left(70\right.$ minutos $\left./ 510^{\circ} \mathrm{C}\right)$, antes da laminação convencional de uma liga de alumínio 7475-T7351. Para tal, duas amostras (com e sem o tratamento térmico) foram laminadas de $12,5 \mathrm{~mm}$ até $3 \mathrm{~mm}$ de espessura, após 13 passes com uma redução aproximada de $10 \%$ por passe. Com base nos resultados obtidos via microscopia óptica (Olympus, modelo GX51) e dureza Vickers (durômetro EMCOTEST, modelo Duravision 300), conclui-se que o tratamento térmico de ressolubilização adotado antes da laminação convencional da liga em estudo, propiciou modificações microestruturais e incremento na dureza quando comparada a amostra laminada sem prévio tratamento térmico de ressolubilização.

Palavras-chave: Laminação, Liga de Alumínio 7475-T7351; Tratamento Térmico de Ressolubilização; Microestrutura; Dureza Vickers.

\section{INFLUENCE OF A RESOLUTION HEAT TREATMENT BEFORE THE ROLLING OF 7475-T7351 ALUMINUM ALLOY}

\begin{abstract}
This study aimed to explore the influence of a resolution heat treatment (70 minutes / $510^{\circ} \mathrm{C}$ ) before the rolling of a 7475-T7351 aluminum alloy. Two samples (one with and one without the heat treatment) were rolled of $12.5 \mathrm{~mm}$ to $3 \mathrm{~mm}$ thickness, after 13 steps with an approximate 10\% reduction per step. After the results from optical microscopy (Olympus, GX51) and Hardness Vickers (EMCOTEST, DuraVision 300), concluded that the resolution heat treatment before the 7475-T7351 alloy rolling (sample TS70-LC), provided a different microstructure and greater hardness than the sample without the heat treatment (sample CR-LC).

Keywords: Rolling, 7475-T7351 aluminum alloy; resolution heat treatment; microstructure; Hardness Vickers.

1 Eng. Metalurgista, M.Sc., Doutorando em Ciência dos Materiais, Instituto Militar de Engenharia, Rio de Janeiro, RJ, Brasil; Professor DI - 1, Centro Federal de Educação Tecnológica Celso Suckow da Fonseca, Angra dos Reis, RJ, Brasil. Contato: saulo brinco@hotmail.com

2 Tecnólogo em Processos Metalúrgicos, Mestrando em Ciência dos Materiais, Instituto Militar de Engenharia, Rio de Janeiro, RJ, Brasil.

3 Química Industrial, M.Sc., Doutoranda em Ciência dos Materiais no CENIMAT/i3N, FCT/UNL, Caparica, Portugal. Bolsista Capes Ciência sem Fronteira Doutorado Pleno.

4 Eng. Metalurgista, D.Sc., Professora Adjunta, Instituto Militar de Engenharia, Rio de Janeiro, RJ, Brasil; e orientadora credenciada no PPGEM/UFF, Volta Redonda, RJ, Brasil.

5 Eng. Metalurgista, D.Sc., Professor Adjunto, Instituto Militar de Engenharia, Rio de Janeiro, RJ, Brasil.
\end{abstract}




\section{INTRODUÇÃO}

Tratamentos térmicos são largamente utilizados em inúmeras aplicações para as mais diversas finalidades. Estes podem ser utilizados apenas para a modificação de propriedades mecânicas de um metal devido a transformação de fase, recristalização de uma matriz encruada, ou então, pode ser utilizado antes de uma operação de conformação mecânica, como por exemplo, a laminação ${ }^{(1)}$.

Dentre as vantagens de se realizar um tratamento térmico antes da laminação, podese destacar a obtenção de um metal/liga mais dúctil, para que este tenha um melhor desempenho durante o processamento em relação a metais/ligas que não tiveram o tratamento térmico (metais encruados).

Um tratamento térmico que pode ser realizado em ligas metálicas (em especial a liga de alumínio 7475, que será utilizada neste trabalho) antes da laminação, é o tratamento térmico de solubilização. Como neste trabalho a liga em estudo já foi submetida a um tratamento térmico de superenvelhecimento -T7351, no qual uma das etapas consiste no tratamento térmico de solubilização da chapa, o tratamento térmico realizado antes da laminação será denominado de ressolubilização.

O tratamento térmico de ressolubilização consiste em aquecer um metal/liga a uma determinada temperatura, e após certo tempo ser atingido, este é resfriado rapidamente até a temperatura ambiente $(2,3)$. Esta operação propicia uma matriz metálica com um maior teor de soluto, condição supersaturada, do que a condição anterior devido a solubilização dos precipitados existentes.

Esta condição se torna interessante para estudo, pois pode alterar os mecanismos operantes durante a laminação da liga de alumínio, como por exemplo, a recuperação/ recristalização, e resultar assim em um material com microestruturas / propriedades mecânicas diferentes do que o metal/liga que não sofreu este tratamento térmico prévio.

O objetivo deste trabalho é verificar a influência de um tratamento térmico de ressolubilização realizado antes da laminação (até $3 \mathrm{~mm}$ de espessura) de uma liga de alumínio 7475-T7351 com espessura inicial de 12,5 mm. Para alcançar tal objetivo, foram utilizados um durômetro (fabricante EMCOTEST, modelo Duravision 300) e um microscópio óptico (Olympus, modelo GX51).

\section{MATERIAL E MÉTODOS}

Como material de partida utilizou-se uma liga de alumínio de nomenclatura 7475T7351, na forma de chapa laminada a quente com uma espessura inicial de $12,5 \mathrm{~mm}$. Antes de realizar a laminação convencional, uma das duas amostras em estudo foi submetida a um tratamento térmico de ressolubilização. Esta amostra foi colocada no interior de um forno (fabricante FDG, modelo 3000) que se encontrava a uma temperatura de $510^{\circ} \mathrm{C}$, durante 70 minutos, e logo após este tempo ser atingido, a mesma foi resfriada até a temperatura ambiente, através da imersão em água sob forte agitação da amostra.

Após 15 minutos do tratamento térmico de ressolubilização, esta amostra juntamente com outra amostra sem ser submetida ao tratamento térmico de ressolubilização, foram laminadas a frio utilizando um laminador piloto do fabricante FENN MFG. Co., modelo D-51710:1973, com uma deformação aproximada de $10 \%$ por passe de laminação até uma espessura de $3 \mathrm{~mm}$ (sendo realizado 13 passes no total em cada amostra). Com base neste processamento as amostras foram nomeadas de CR-LC (amostra sem tratamento térmico prévio e submetida a laminação convencional) e 
TS70-LC (amostra submetida ao tratamento térmico de solubilização e submetida a laminação convencional), além da amostra como recebida (CR).

Para a visualização da microestrutura das amostras CR, CR-LC e TS70-LC, as mesmas foram lixadas com lixas de carbeto de silício de granulometrias de 220 a 1200 mesh, polidas eletroliticamente com uma solução de $1 / 3$ de ácido nítrico $\left(\mathrm{HNO}_{3}\right)$ e $2 / 3$ de Metanol, utilizando 15 volts e 15 segundos de polimento.

Após o polimento, com intuito de revelar os contornos dos grãos, as amostras foram imersas em uma solução contendo $4 \%$ de $\mathrm{NaOH}$ e $96 \%$ de $\mathrm{H}_{2} \mathrm{O}$, em peso, durante aproximadamente 60 segundos, com subsequente imersão em Nital 5\% (5\% de Ácido Nítrico + 95\% de Álcool Etílico 98,9\% PA, ambos em volume) durante aproximadamente 3 segundos, logo em seguida as amostras foram lavadas com água corrente, álcool e secadas com jato de ar quente. Esta sequência foi repetida sete vezes.

Para a visualização da microestrutura das amostras foi utilizado um microscópio óptico do fabricante Olympus, modelo GX51, acoplado a uma câmera digital colorida (marca Olympus, modelo DP25), controlada pelo software de aquisição e análise de imagens OLYMPUS Analysis.

Para a análise de dureza Vickers (durômetro digital, fabricante EMCOTEST, modelo Duravision 300) as amostras CR, CR-LC e TS70-LC tiveram a seção transversal a direção de laminação lixadas em uma lixadeira rotativa manual com lixas de carbeto de silício de 220, 320, 500, 800, 1000, 1200 e 2500 mesh. As análises de dureza Vickers foram realizadas com uma carga de $50 \mathrm{kgf}$, durante um tempo de permanência de 15 segundos nas amostras.

$\mathrm{Na}$ amostra $\mathrm{CR}$ foram realizadas 5 medições em regiões associadas a 1/4, 1/2 e 3/4 da espessura da chapa $(12,5 \mathrm{~mm})$ e para as amostras CR-LC e TS70-LC foram realizadas 10 medições a $1 / 2$ espessura das chapas $(3 \mathrm{~mm})$.

\section{RESULTADOS E DISCUSSÃO}

Diniz (2016) em seu estudo, realizou a caracterização via microscopia ótica e dureza Vickers da liga de alumínio 7575-T7351, na condição de como recebida (CR) e submetida ao tratamento térmico de solubilização com tempo de encharque de 70 minutos (TS70). Com base neste estudo, pode-se observar que após o tratamento térmico (amostra TS70) não houve diferenças significativas em relação a amostra CR. $\mathrm{Na}$ Figura 1 são apresentadas as microestruturas ao longo da espessura das amostras (a) CR-LC e (b) TS70-LC após o polimento eletrolítico com um aumento de 50x. Na Figura 1, "PG" indicam os precipitados grosseiros.

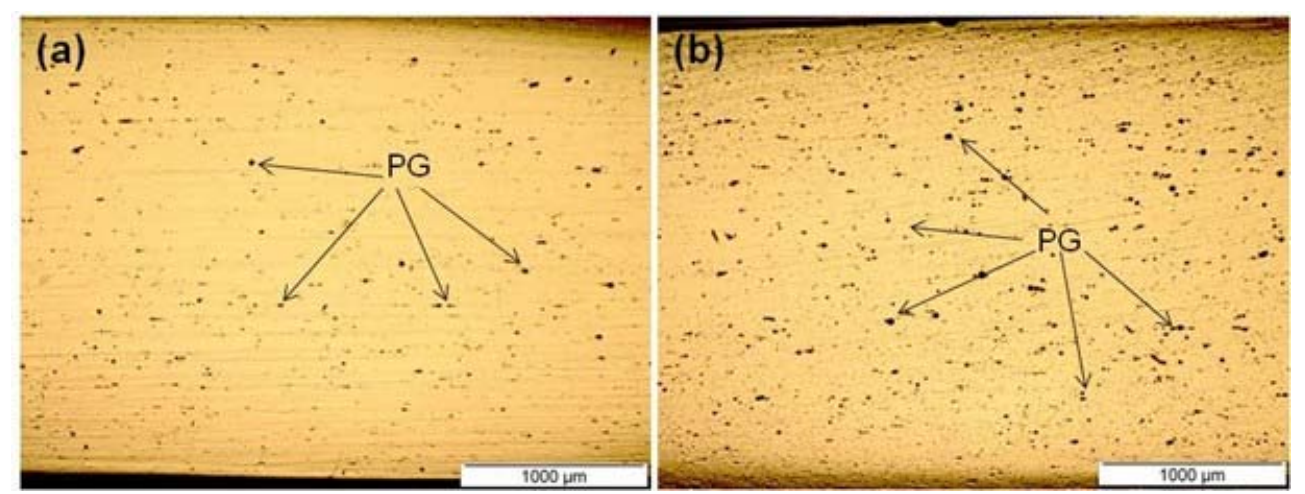

Figura 1. Microestruturas das amostras (a) CR-LC e (b) TS70-LC. Polimento eletrolítico, aumento de $50 x$, sem ataque. 
Como pode ser observado na Figura 1, evidencia-se frações volumétricas de precipitados grosseiros distintos entre as amostras CR-LC e TS70-LC, no qual a amostra TS70-LC apresenta uma aparente maior fração volumétrica de precipitados do que a amostra CR-LC.

Nas Figuras 2 e 3 são apresentadas as microestruturas das amostras CR-LC (a, c, e) e TS70-LC (b, d, f) visualizadas à $1 / 4$ da espessura $((a)$ e (b)), $1 / 2$ espessura $((c)$ e (d)) e $3 / 4$ da espessura ((e) e (f)), com um aumento de 200x - Figura 2 e 1000x - Figura 3. Nas Figuras 2 e 3, "GC" indicam grãos com colorações mais claras, "GE" grãos com colorações mais escuras, "PG" indica os precipitados grosseiros, "PI" precipitados com tamanho intermediário e "PD" precipitado de menor dimensão.
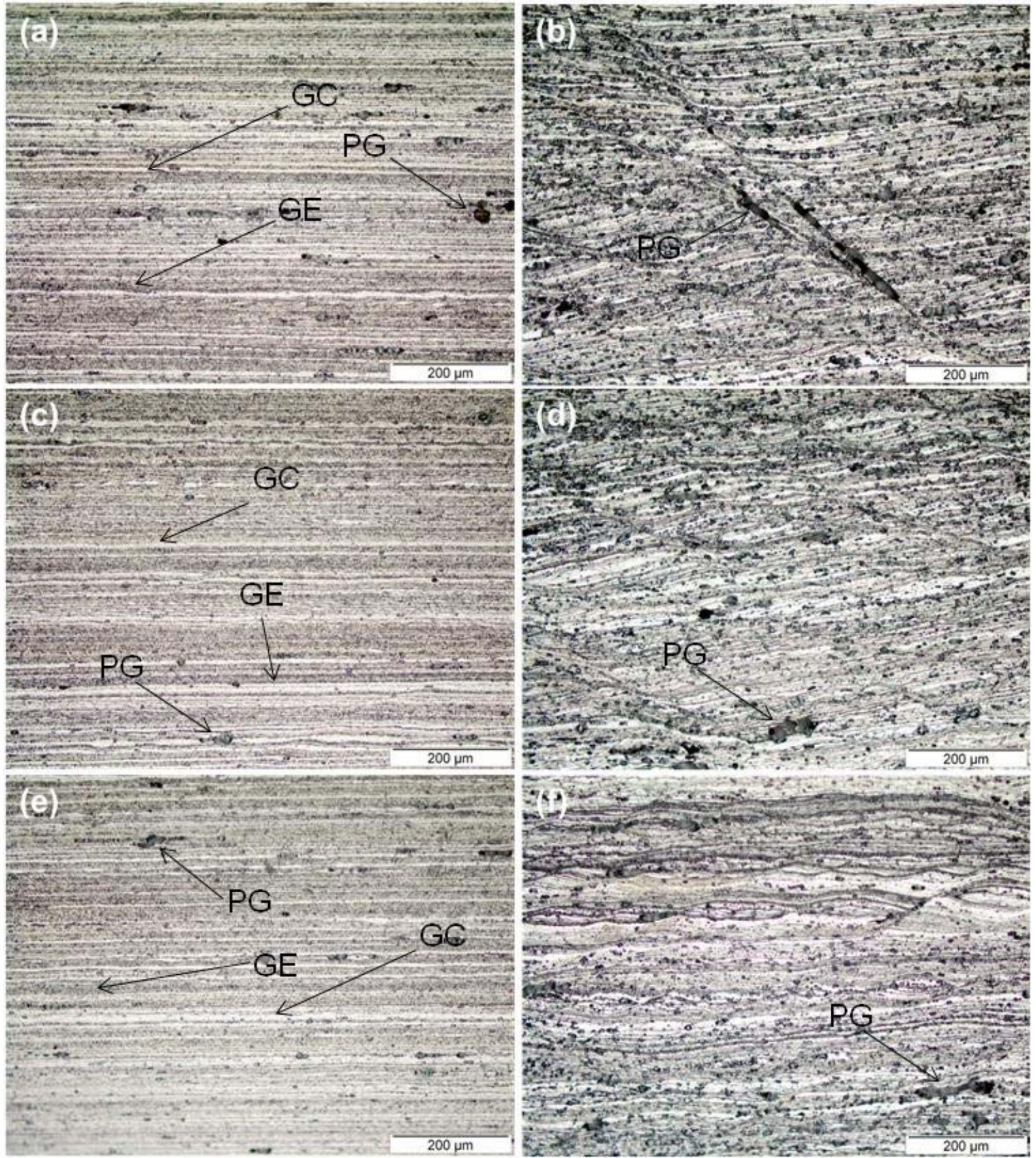

Figura 2. Microestrutura das amostras CR-LC (a, c, e) e TS70-LC (b, d, f) visualizadas à $1 / 4$ da espessura ((a) e (b)), $1 / 2$ espessura ((c) e (d)) e 3/4 da espessura ((e) e (f)), com um aumento de $200 x$. Microscopia óptica, amostras atacadas. 

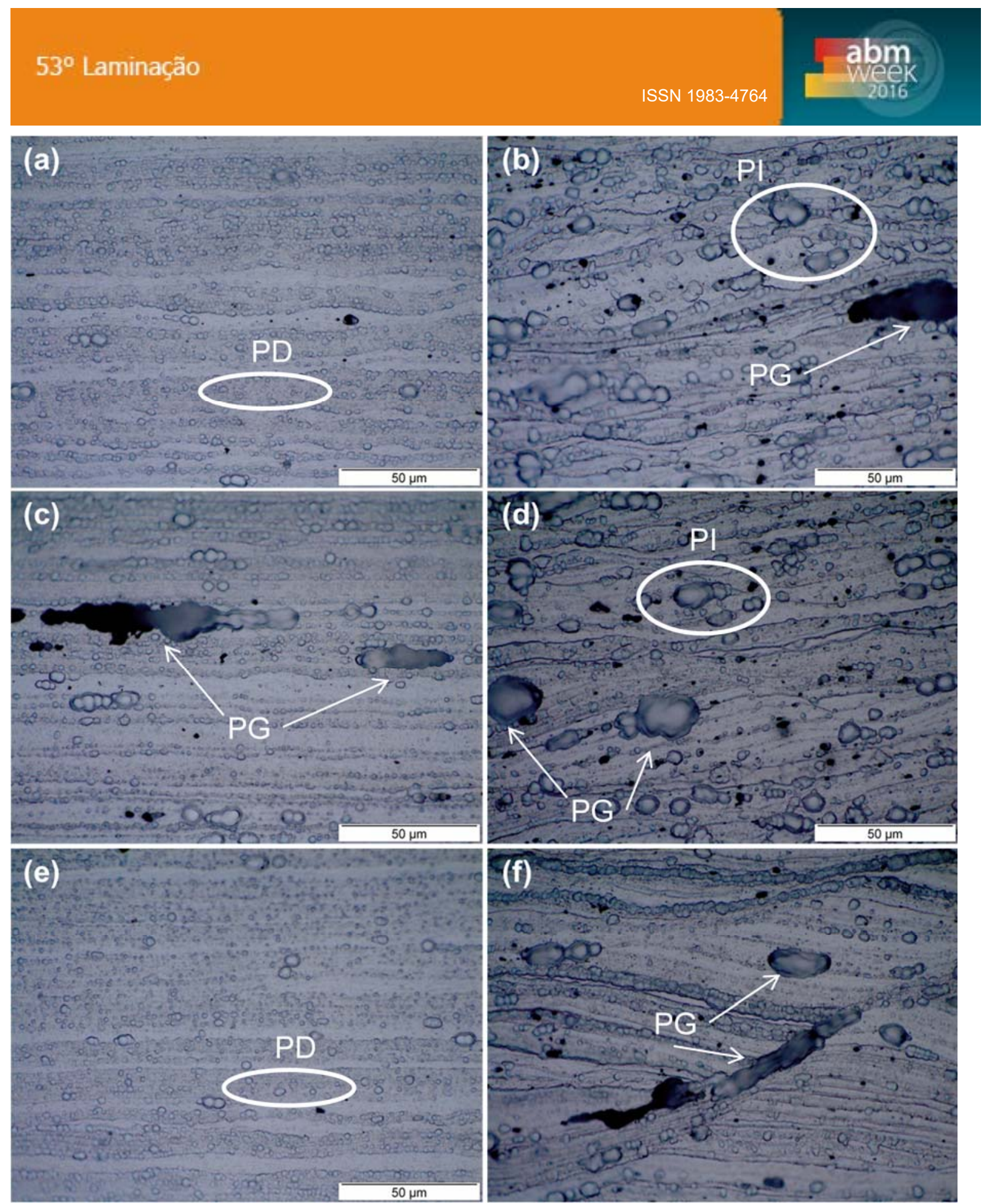

Figura 3. Microestrutura das amostras CR-LC (a, c, e) e TS70-LC (b, d, f) visualizadas à $1 / 4$ da espessura ((a) e (b)), $1 / 2$ espessura ((c) e (d)) e 3/4 da espessura ((e) e (f)), com um aumento de 1000x. Microscopia óptica, amostras atacadas.

Conforme observado na Figura 2, observa-se diferenças significativas entre as microestruturas das amostras CR-LC e TS70-LC. A amostra CR-LC apresenta grãos com coloração mais clara e grãos com coloração mais escura, e outros grãos que tem uma coloração intermediária. Todos os grãos estão alongados na direção de laminação. Há também a presença de precipitados grosseiros (indicados por "PG"). Já a amostra TS70-LC, não é possível observar os grãos claros e escuros com tanta facilidade como na amostra CR-LC. Também pode-se observar que os grãos não estão claramente alongados na direção de laminação como a amostra CR-LC, no qual apresentam distorções.

Na Figura 3 com um maior aumento, é destacado a diferença microestrutural entre as amostras CR-LC e TS70-LC, no qual a amostra CR-LC apresenta precipitados de 
menor dimensão (indicado por "PD") e a amostra TS70-LC apresenta uma fração volumétrica muito maior de precipitados com tamanho intermediário (indicado por "Pl").

Estas diferenças microestruturais entre as amostras em estudo podem resultar em valores de dureza diferentes. Na Tabela 1 são apresentados os valores de dureza Vickers das amostras CR, CR-LC e TS70-LC.

Tabela 1. Dureza Vickers das amostras CR ( $1 / 4,1 / 2$ e $3 / 4$ da espessura), CR-LC (1/2 espessura) e TS70LC ( $1 / 2$ espessura). Carga de $50 \mathrm{kgf}$.

\begin{tabular}{|c|c|c|c|c|c|}
\hline Amostra & Posição & Média & $\begin{array}{c}\text { Desvio } \\
\text { Padrão }\end{array}$ & Erro & $\begin{array}{c}\text { Porcentagem de incremento } \\
\text { da dureza Vickers em } \\
\text { relação a amostra CR-1/2 } \\
\text { espessura }\end{array}$ \\
\hline \multirow{2}{*}{ CR } & $\mathbf{1 / 4}$ espessura & 163,6 & 2,61 & 3,24 & - \\
\cline { 2 - 6 } & $\mathbf{1 / 2}$ espessura & 158,2 & 3,49 & 4,34 & - \\
\cline { 2 - 6 } & $\mathbf{3 / 4}$ espessura & 160,2 & 3,49 & 4,34 & - \\
\hline CR-LC & $\mathbf{1 / 2}$ espessura & 170,50 & 0,71 & 0,51 & $7,77 \%$ \\
\hline TS70-LC & $\mathbf{1 / 2}$ espessura & 194,60 & 1,52 & 1,08 & $21,47 \%$ \\
\hline
\end{tabular}

Conforme apresentado na Tabela 1, a amostra CR apresentou uma dureza Vickers média de 163,6 a 1/4 da espessura, 158,2 a 1/2 espessura e 160,2 a $3 / 4$ da espessura. Após a laminação convencional da amostra sem tratamento térmico e da amostra que foi submetida previamente ao tratamento térmico de solubilização, amostras CR-LC e TS70-LC, respectivamente, pode-se observar valores de dureza diferentes entre si.

A amostra CR-LC apresentou um valor de dureza médio de 170,50 HV, ou seja, um incremento de $12,3 \mathrm{HV}$ em relação os valores medidos a $1 / 2$ da espessura na amostra CR. Este incremento representa apenas $7,77 \%$, no qual é um valor muito pequeno para uma amostra que foi laminada de uma espessura inicial de 12,5 para $3 \mathrm{~mm}$. Tal comportamento pode ser explicado devido a recuperação dinâmica que ocorreu durante a laminação.

A amostra TS70-LC apresentou uma dureza de 194,60 HV, no qual teve um incremento de $36,40 \mathrm{HV}$, que representa $21,47 \%$ de incremento de dureza em relação a dureza da amostra CR medida a $1 / 2$ espessura.

Como observado, a realização de um tratamento térmico de solubilização antes da laminação, foi o suficiente para obter uma microestrutura diferente da microestrutura do material apenas laminado, e consequentemente um incremento maior de dureza após a laminação.

\section{CONCLUSÃO}

De acordo com o estudo realizado pode-se concluir que a realização de um tratamento térmico de ressolubilização com tempo de encharque de 70 minutos a $510^{\circ} \mathrm{C}$ antes da laminação convencional de uma liga de alumínio 7475-T7351 (amostra TS70-LC), proporcionou uma microestrutura diferente e consequentemente uma maior dureza do que a amostra sem tratamento térmico e laminada convencionalmente (CR-LC). 


\section{Agradecimentos}

Os autores agradecem a CAPES pela bolsa de doutorado do autor Saulo Brinco Diniz e ao Instituto SENAI de Tecnologia - Solda, pela utilização do durômetro digital EMCOTEST- Duravision 300 e microscópio óptico Olympus - GX51 / câmera digital colorida Olympus - DP25. A autora A. S. Paula agradece ao CNPq pela bolsa de produtividade nível 2 (Processo 307798/2015-1).

\section{REFERÊNCIAS}

1 Bresciani Filho E, Zavaglia CAC, Button ST, Gomes EN, Fernando AC. Conformação Plástica dos Metais. $4^{\circ}$ Edição Revisada e Ampliada. São Paulo: Editora da Unicamp, 1991, 352 f. Volume Único.

2 ASM Handook. Heat Treating. ASM International: volume 4, 1991

3 ASM Handook.Properties and Selection: Nonferrous Alloys and Special-Purpose Materials. ASM International: volume 2, 1990.

4 Diniz SB, Paula AS, Brandão LPM. Tratamento Térmico de Ressolubilização em uma Liga de Alumínio 7475-T7351. 71 Congresso Anual da ABM, In: ABM Week, 2016. 